NASA Technıcal Memorandum 86008

NASA-TM-86008 19840026938

\title{
Enthalpy Damping for the Steady Euler Equations
}

\section{Dennis Jespersen}

October 1984

\section{HePRY RPP \\ แッンะ 1984}

LANGLEY RESEARCH CENTER LIBRARY, NASA

HA:IPTON, VIRGINIA 
NASA Technical Memorandum 86008

\section{Enthalpy Damping for the Steady Euler Equations}

Dennıs Jespersen, Ames Research Center, Moffett Field, Calıfornıa 


\title{
Enthalpy Damplng for the Steady Euler Equations
}

\author{
DENNIS C. JESPERSEN ${ }^{2}$ \\ MS 202A-1, NASA Ames Research Center \\ Moffett Field, CA 94035 USA
}

\begin{abstract}
SUMMARY. For inviscid steady How problems where the entlualpy is constant at steady state, it has been proposed by Jameson, Schmidt, and Turkel to use the difference between the local enthalpy and the steady state enthalpy as a dnving term to accelerate convergence of iterative schemes This adea is analyzed here, both on the level of the partial differential equation and on the level of a particular finite difference scheme It is shown that for the two-dimensional unsteady Euler equations, a hyperbolic system with ergenvalues on the imaginary axis, there is no enthalpy damping strategy which can move all the ergenvalues into the open left half plane. For the numencal scheme, however, the analysis shows and examples verify that enthalpy damping can be effectuve in accelerating convergence to steady state.
\end{abstract}

\section{INTRODUCTION}

Rapidly convergent numerical schemes for steady state solutions of the inviscid compressible fluid dynamics equations, the Euler equations, have been studied intensively in the last few years. Algorithms and codes have been developed by Jameson, Schmidt, and Turkel (non-multigrid) (Ref 1); Jameson (multignd) (Ref 2), Rizzi (Ref 3); Pullıam (Ref. 4), Lerat, Sidès, and Daru (Ref 5), Johnson (Ref. 6); Nı (Ref. 7); Agarwal and Deese (Ref 8); Osher and Chakravarthy (Ref. 9), Mulder and van Leer (Ref 10), and by Buning and Steger (Ref 11), among others One of the most successful and widely used algorithms is embodied in the FLO52 codes of A Jameson The algorithm used for the Euler equations in these codes is based on an explcit multıstage time-stepping scheme, with (for steady state problems) acceleration devices such as local tıme step selectıon, implicit residual averaging, multigrid, and enthalpy damping It is the purpose of this paper to provide an analysis of enthalpy damping for the two-dimensional Euler equations

The origmal derivation of enthalpy damping by Jameson, Schmidt, and Turkel (Ref. 1) was based on reducing the unsteady Euler equations to the unsteady potential equation (a wave equation), introducing frictional damping to modify the equation to the telegraph equation, and translating the new equation back to the Euler equations The next section begins by reviewing this derivation It can be verified that enthalpy damping is effective in enhancing convergence for the Euler equations, and an example calculation is included.

The two-dimensional Euler equations with enthalpy damping are studied in section 3. The Euler equations are a system of partial differential equations of the form

$$
w_{t}+\partial_{x} \mathcal{E}(w)+\partial_{y} \mathcal{F}(w)+\alpha \mathcal{H}(w)=0
$$

where the enthalpy damping is embodied in the undifferentiated term $H$, and $\alpha$ determines the strength of the damping term We linearize $\left(\begin{array}{ll}1 & 1\end{array}\right)$ around a state $w_{0}$ with constant enthalpy, obtaining a linear variable coefficient system

$$
w_{t}+\partial_{x}\left(A\left(w_{0}(x, y)\right) w\right)+\partial_{y}\left(B\left(w_{0}(x, y)\right) w\right)+\alpha C\left(w_{0}(x, y)\right) w=0
$$

where $w$ is the perturbation from $w_{0}$ and $A=\partial \mathcal{E} / \partial w, B=\partial \mathcal{F} / \partial w, C=\partial H / \partial w$. We study the frozen coefficient problem

$$
w_{t}+A \partial_{x} w+B \partial_{y} w+\alpha C w=0
$$

Fourier transforming ( 13 ) gives the system

$$
\frac{d \hat{w}}{d t}+1\left(\omega_{1} A+\omega_{2} B\right) \hat{w}+\alpha C \hat{w}=0
$$

where $w(x, y, t)=e^{t\left(\omega_{1} x+\omega_{2} y\right)} \hat{w}(t)$. For $\alpha=0$, the system $(13)$ is hyperbolic and all solutions $\hat{w}$ of $(14)$ are of the form $\widehat{w}=\sum, e^{\lambda, t} \hat{w},(0)$ where $\lambda$ is purely imaginary. For $\alpha>0$ the natural conjecture is that the enthalpy damping helps by pulling the $\lambda$, into the left half of the complex plane. We show that of the four eigenvalues $\lambda$, for any pair $\left(\omega_{1}, \omega_{2}\right)$, one eigenvalue always remains on the imaginary axis, so the system $(13)$ does not have the property

\footnotetext{
'Research Scientist, Computational Fluid Dynamics Branch
} 
that all its solutions decay to zero. The analysis also suggests a form of the enthalpy damping term for the energy equation more natural than that of Jameson, Schmidt, and Turkel (Ref. 1).

If the Euler equations at the partial differential equation level do not have decaying solutions when enthalpy damping is in effect, why do the numerical results show that enthalpy damping is useful? This question is addressed in section 4. To get a flavor of the idea, consider the system of ordinary differential equations

$$
\frac{d u}{d t}+A u=0
$$

where the complex matrix $A$ is assumed to be diagonalizable with all its eigenvalues on the imaginary axis, say at s $\lambda_{1}, i \lambda_{2}, \ldots$, with $0<\lambda_{1}<\lambda_{2}<\ldots$. Solutions to (15) do not decay to zero as $t \rightarrow \infty$. However, if (15) is integrated with the implicit Euler method

$$
u_{n+1}-u_{n}+\Delta t A u_{n+1}=0
$$

the numerical solution $u_{n}$ will go to zero as $n \rightarrow \infty$ because the eigenvalues of $A$ are contaned in the interior of the stability region of the method Furthermore, the eigenvector associated with the eigenvalue $i \lambda_{1}$ will decay slowest because its damping factor is closest to 1 Thus if the matrix $A$ is somehow modified so that the eigenvalue $\lambda_{1}$ moves to the left, such that the modified eigenvalue is farther from 1 in the complex plane than the original eigenvalue, and such that all the other elgenvalues are unchanged, then the numerical solution of the modified system will decay to zero faster than the numerical solution of the original system In section 4 we attempt to show that roughly the same phenomenon occurs with the Euler equations and an explcit multistage integration scheme ( $\mathrm{e}$, the eigenvectors obstructing the convergence are associated with eigenvalues which are moved to the left when enthalpy damping is used) Thus, even though one of the eigenvalues does not move off the imagnary axis, the convergence rate can still improve An attempt is made to quantify the improvement in convergence rate and to find an optimal damping parameter $\alpha$

The major omission of this work is the neglect of numerical dissipation. The numerical scheme is actually an approximation not to $\left(\begin{array}{ll}1 & 1\end{array}\right)$, but to

$$
w_{t}+\partial_{x} \mathcal{E}(w)+\partial_{y} \mathcal{F}(w)+\alpha \mathcal{H}(w)+\epsilon D(w)=0
$$

where $D$ is a dissipation operator (e $g$, a blend of second- and fourth-order dissipation terms) The inclusion of a dissipation term makes the analysis much more cumbersome, a point which will be touched on again If an analysis including the dissipation term could be carned out, results more relevant to the performance of the computer codes could be obtained

The author bas had the benefit of several llluminating discussions with Elı Turkel in the course of this work Many of the algebraic computations in sections 3 and 4 were carried out with vaxıma, the VAX version of Macsyma (Ref. 12) Some of the computations would have been extremely difficult or impossible without vaxıma.

\section{Derivation of Enthalpy Damping}

This section reviews the derivation of enthalpy damping via the unsteady potential equation and telegraph equation as originally sketched in Jameson, Schmidt, and Turkel (Ref 1) The two-dimensional Euler equations in conservation form for a perfect gas may be written

$$
\begin{array}{r}
\rho_{t}+(\rho u)_{x}+(\rho v)_{y}=0 \\
(\rho u)_{t}+\left(\rho u^{2}+p\right)_{x}+(\rho u)_{y}=0 \\
(\rho v)_{t}+(\rho u v)_{x}+\left(\rho v^{2}+p\right)_{y}=0 \\
(\rho E)_{t}+(\rho u H)_{x}+(\rho v H)_{y}=0
\end{array}
$$

where $\rho$ is the density, $u$ and $v$ are Cartesian velocity components, $E$ is total energy per unit mass, $p$ is pressure, and $H$ is the enthalpy, defined by

$$
H=E+p / \rho
$$


One can verify that

$$
\frac{D}{D t} H=\frac{1}{\rho} \frac{\partial p}{\partial t}
$$

where $D / D t$ is the material derivative $D / D t:=\partial / \partial t+v \cdot \nabla$. (The notation $a=b$ means $a$ is defined as $b$ ) Hence in steady flow $H$ is constant along streamlnes, and if flow conditions are uniform at upstream infinity, then $H$ is everywhere constant at steady state.

In nonconservative form the Euler equations may be written

$$
\begin{aligned}
\rho_{t}+u \rho_{x}+v \rho_{y}+\rho\left(u_{x}+v_{y}\right) & =0 \\
u_{t}+u u_{x}+v u_{y}+p_{x} / \rho & =0 \\
v_{t}+u v_{x}+v v_{y}+p_{y} / \rho & =0 \\
E_{t}+u E_{x}+v E_{y}+\frac{p}{\rho}\left(u_{x}+v_{y}\right)+\frac{1}{\rho}\left(u p_{x}+v p_{y}\right) & =0
\end{aligned}
$$

The fourth equation here can be replaced by the transport equation for entropy

$$
S_{t}+u S_{x}+v S_{y}=0
$$

The choice of the fourth equation affects the form of enthalpy damping that is derived

If the flow is barotropic (i e., $p=p(\rho)$ ) one can show by differentiating the $x$-momentum equation with respect to $y$, the $y$-momentum equation with respect to $x$, and subtracting the latter from the former, that the vorticity $\omega=v_{x}-u_{y}$ satisfies

$$
\omega_{t}+(\omega u)_{x}+(\omega v)_{y}=0
$$

With this and the use of the continuty equation one can show that

$$
\frac{\partial(\omega / \rho)}{\partial t}+u \partial_{x}(\omega / \rho)+v \partial_{y}(\omega / \rho)=0
$$

Thus if the flud is irrotational (i e , $\omega=0$ ) at time $t=0$ then it is irrotational for all $t>0$. For an irrotational furd a velocity potential $\phi$ may be introduced, where $\phi_{x}=u$ and $\phi_{y}=v$ Then the momentum equations may be integrated to give the unsteady Bernoulh equation

$$
\frac{\partial \phi}{\partial t}+\frac{u^{2}+v^{2}}{2}+\int \frac{d p}{\rho}=G(t)
$$

where $G$ is an arbitrary function of $t$ If the flow is homentropic (entropy everywhere constant), then we have $p=A \rho^{\gamma}$ and $\int d p / \rho=\gamma p /[(\gamma-1) \rho]$ For an ideal gas we have $p=(\gamma-1) \rho\left[E-\left(u^{2}+v^{2}\right) / 2\right]$ where $\gamma$ is the ratio of specific heats Hence

$$
H=E+p / \rho=\frac{\gamma}{\gamma-1} \frac{p}{\rho}+\frac{u^{2}+v^{2}}{2}
$$

and the unsteady Bernoull equation becomes

$$
\frac{\partial \phi}{\partial t}+H=G(t)
$$

For extenor flow problems, if the conditions are uniform at infinity, this becomes

$$
\frac{\partial \phi}{\partial t}+H=H_{\infty}
$$

where $H_{\infty}$ is the enthalpy at infinity

An alternate form of the unsteady Bernoull equation (2 5) is

$$
\phi_{t}+\frac{\phi_{x}^{2}+\phi_{y}^{2}}{2}+\frac{\gamma}{\gamma-1} A \rho^{\gamma-1}=H_{\infty}
$$


To obtain the unsteady potential equation, this equation is differentiated with respect to $t, x$, and $y$, and solved for $\rho_{t}, \rho_{x}, \rho_{y}$. These expressions for $\rho_{t}, \rho_{x}$, and $\rho_{y}$ are substituted into the mass conservation equation. Using the relation giving the speed of sound as $c^{2}=\gamma p / \rho$, one obtains the unsteady potential equation

$$
\phi_{t t}+2 u \phi_{x t}+2 v \phi_{y t}=\left(c^{2}-u^{2}\right) \phi_{x x}-2 u v \phi_{x y}+\left(c^{2}-v^{2}\right) \phi_{y y}
$$

For constant $u$ and $v$ the change of coordinates $\xi=x-u t, \eta=y-v t$ transforms this to the wave equation

$$
\phi_{t t}=c^{2}\left(\phi_{\xi \xi}+\phi_{\eta \eta}\right)
$$

A related equation (Ref. 13) is the telegraph equation

$$
\phi_{t t}+\alpha \phi_{t}=c^{2}\left(\phi_{\xi \xi}+\phi_{\eta \eta}\right)
$$

For $\alpha>0$ the solutions of this equation decay as $t \rightarrow \infty$. Transforming this equation back via $x=\xi+u t, y=\eta+v t$ we obtain the damped version of (26),

$$
\phi_{t t}+2 u \phi_{x t}+2 v \phi_{y t}+\alpha \phi_{t}=\left(c^{2}-u^{2}\right) \phi_{x x}-2 u v \phi_{x y}+\left(c^{2}-v^{2}\right) \phi_{y y}
$$

This is the equation one would have obtaned from the mass conservation equation if the mass conservation equation had been

$$
\rho_{t}+u \rho_{x}+v \rho_{y}+\rho\left(u_{x}+v_{y}\right)=\alpha \rho \phi_{t}
$$

Now, $\phi$ is unknown, but the unsteady Bernoulli equation (25) allows us to replace $\phi_{t}$ by $H_{\infty}-H$, obtaining the modified mass conservation equation

$$
\rho_{t}+u \rho_{x}+v \rho_{y}+\rho\left(u_{x}+v_{y}\right)+\alpha \rho\left(H-H_{\infty}\right)=0
$$

Using this in place of the first equation of (22) and recombining into conservation form, one obtains the system of equations

$$
\begin{aligned}
\rho_{t}+(\rho u)_{x}+(\rho v)_{y}+\alpha \rho\left(H-H_{\infty}\right) & =0 \\
(\rho u)_{t}+\left(\rho u^{2}+p\right)_{x}+(\rho u v)_{y}+\alpha \rho u\left(H-H_{\infty}\right) & =0 \\
(\rho v)_{t}+(\rho u v)_{x}+\left(\rho v^{2}+p\right)_{y}+\alpha \rho v\left(H-H_{\infty}\right) & =0 \\
(\rho E)_{t}+(\rho u H)_{x}+(\rho v H)_{y}+\alpha \rho E\left(H-H_{\infty}\right) & =0
\end{aligned}
$$

If the entropy equation is used as the fourth equation of $(22)$, recombining into conservation form produces a different fourth equation in (29) Then (29) is replaced by

$$
\begin{aligned}
\rho_{t}+(\rho u)_{x}+(\rho v)_{y}+\alpha \rho\left(H-H_{\infty}\right) & =0 \\
(\rho u)_{t}+\left(\rho u^{2}+p\right)_{x}+(\rho u v)_{y}+\alpha \rho u\left(H-H_{\infty}\right) & =0 \\
(\rho v)_{t}+(\rho u v)_{x}+\left(\rho v^{2}+p\right)_{y}+\alpha \rho v\left(H-H_{\infty}\right) & =0 \\
(\rho E)_{t}+(\rho u H)_{x}+(\rho v H)_{y}+\alpha \rho H\left(H-H_{\infty}\right) & =0
\end{aligned}
$$

In the next section we will analyze a general form of enthalpy damping which includes both (29) and (2 10).

In summary, for subsonic irrotational flow, systems (29) and (2 10) give rise (locally) to the telegraph equation while the system $(21)$ gives rise (locally) to the wave equation. Furthermore, we may hope that steady states of $(29)$ and $(210)$ are steady states of $(21)$, and vice versa. It is clear that any steady state of $(21)$ is also a steady state of (2.9) and (2 10) (since steady states of (2 1) have constant enthalpy). It is not clear that steady states of (2 9) are steady states of (2 1). It is true, however, that steady solutions of $(210)$ have enthalpy constant along streamlınes, hence if flow conditions are unform at upstream infinty then steady solutions of $(210)$ have enthalpy constant every where, hence steady solutions of $(210)$ are also steady solutions of $(21)$. To see that steady solutions of $(210)$ have enthalpy constant along streamlines, multiply the first equation of $(210)$ by $H$ and subtract the result from the fourth equation of (210). If the time derivatives are zero one finds that $u H_{x}+v H_{y}=0$, hence $H$ is constant along streamlines 
In place of the fourth equation of (2.9) we can use

$$
\left(\rho\left(E-H_{\infty}\right)\right)_{t}+\left(\rho u\left(H-H_{\infty}\right)\right)_{x}+\left(\rho v\left(H-H_{\infty}\right)\right)_{y}+\alpha \rho\left(E-H_{\infty}\right)\left(H-H_{\infty}\right)=0
$$

which is obtained by multiplying the first equation of (2.9) by $H_{\infty}$ and subtracting from the last equation of (2.9).

It is suggested by Jameson, Schmidt, and Turkel (Ref. 1) that a forcing term $\alpha \rho H\left(H-H_{\infty}\right)$ in the fourth equation of (29) "can be destabilizing," and it is suggested that this term be replaced with $\alpha\left(H-H_{\infty}\right)$. The computer codes seen by this author use a forcing term $\alpha \rho\left(H-H_{\infty}\right)$ in (2 11); i.e., $E-H_{\infty}$ is replaced by 1 in the fourth term of (2 11). Thes ad hoc fix seems undesirable, if only on dimensional grounds. One of the purposes of this paper is to rationally dernve a reasonable form for the forcing term in the energy equation, thereby clearing up some of the confusion that seems to exist.

Figure 21 shows a comparison of a Euler equation calculation with and without enthalpy damping. The code used was FLO52R (supplied by $A$ Jameson) with the multigrid and implicit residual averaging options turned off. The intial solution was a partially converged solution obtained by grid continuation (interpolation from a coarser grid) The enthalpy damping factor $\alpha$ was 005 The convergence rate improved from 09966 without enthalpy damping to 09954 with enthalpy damping. Jameson, Schmidt, and Turkel (Ref. 1) show an example of improvement in convergence rate from 09909 to 09863 . The greater gain reported there may perhaps be due to the smaller number of grid points $(128 \times 32)$ or other mesh differences, differences in the boundary conditions, or differences in implementation of the numerical algorithm

\section{3 analysis of Enthalpy Damping for the Differential Equation}

Consider the system of partial differential equations

$$
w_{t}+\partial_{x} \dot{E}(w)+\partial_{y} \xi(w)+\alpha \mathcal{H}(w)=0
$$

where

$$
\begin{aligned}
w & =(\rho, \rho u, \rho v, \rho E)^{T} \\
\mathcal{E} & =\left(\rho u, \rho u^{2}+p, \rho u v, \rho u H\right)^{\mathrm{T}} \\
\mathcal{F} & =\left(\rho v, \rho u v, \rho v^{2}+p, \rho v H\right)^{\mathrm{T}} \\
\mathcal{H} & =\left(\rho\left(H-H_{\infty}\right), \rho u\left(H-H_{\infty}\right), \rho v\left(H-H_{\infty}\right), \rho \tilde{h}_{4}\left(H-H_{\infty}\right)\right)^{T}
\end{aligned}
$$

Here we have taken a farly general form of the fourth component of $H$, with $\tilde{h}_{4}=\tilde{h}_{1}(w)$ On dimensional grounds, $\tilde{h}_{1}$ should have the same units as $E$

The first step is to linearize about a steady solution $w_{0}$, and since we are interested in steady solutions with constant enthalpy, we will assume $w_{0}$ has constant enthalpy The first-order terms in the equation for the variation $w_{0}+w$ then give the linear variable coefficient equation for $w$

$$
\partial_{\imath} w+\partial_{x}\left(A\left(w_{0}(x, y)\right) w\right)+\partial_{y}\left(B\left(w_{0}(x, y)\right) w\right)+\alpha C\left(w_{0}(x, y)\right) w=0
$$

where $A, B, C$ are the Jacobian matrices of $\mathcal{E}, \mathcal{F}$, and $\mathcal{H}$, respectıvely We will study this variable coefficient system by freezing the coefficients at an arbitrary $\left(x_{0}, y_{0}\right)$ This gives the constant coefficient problem

$$
\partial_{t} w+A \partial_{x} w+B \partial_{y} w+\alpha C w=0
$$

The justification for this reduction is the conjecture that if all the constant coefficient problems (3 4) have $w \rightarrow 0$ as $t \rightarrow \infty$, then the variable coefficient problem (33) has $w \rightarrow 0$ as $t \rightarrow \infty$, and that if the variable coefficient problem (33) has $w \rightarrow 0$ as $t \rightarrow \infty$ then the nonlinear problem is (locally) asymptotically stable at a steady state with constant enthalpy This author is not aware of any theorems which rigorously quantify these conjectures

We will analyze ( 3 4) by Fourier methods, take

$$
w(x, y, t)=e^{\imath\left(\omega_{1} x+\omega_{2} y\right)} \hat{w}(t)
$$




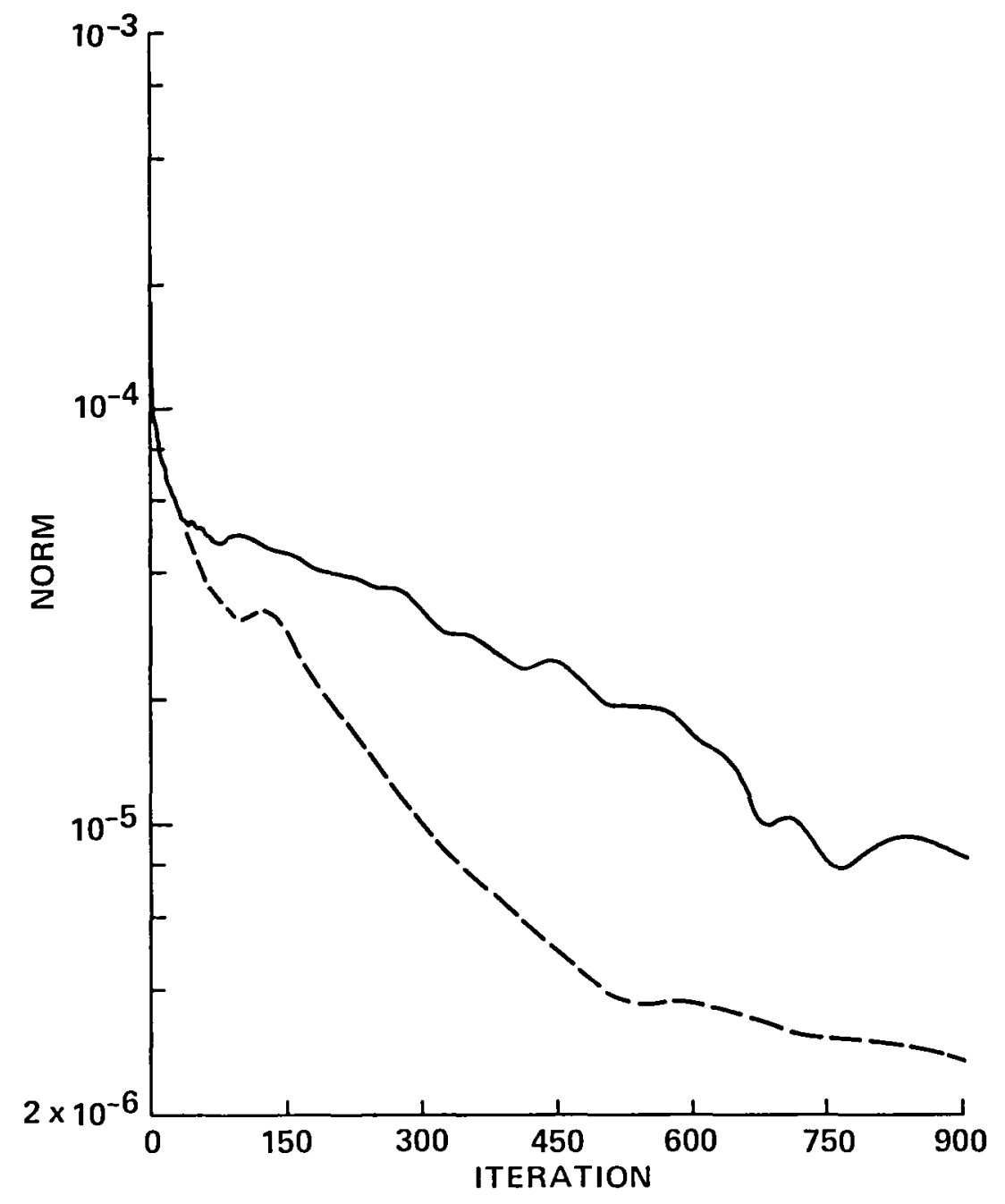

Figure $21 L_{2}$ norm of residual in density equation for FLO52R without enthalpy damping (solsd line) and with enthalpy damping (dashed line) NACA0012 arfoll, $M_{\infty}=08$, angle of attack $=125^{\circ}, 192 \times 32$ mesh, $\mathrm{CFL}=2$, four-stage method, coefficients $(1 / 4,1 / 3,1 / 2,1)$, two dissipation evaluations Iteration started with partially converged solution.

and substitute in (3 4) to obtain the ordinary differential equation

$$
\frac{d \hat{w}}{d l}+\left[\imath\left(\omega_{1} A+\omega_{2} B\right)+\alpha C\right] \widehat{w}=0
$$

Here take $\widehat{w}=e^{z t} w$ where $z$ is complex and $w$ is a constant four-vector. This gives

$$
\left(z I+i\left(\omega_{1} A+\omega_{2} B\right)+\alpha C\right) w=0
$$

If all the zeros of the polynomial $p(z):=\operatorname{det}\left(z I+i\left(\omega_{1} A+\omega_{2} B\right)+\alpha C\right)$ lie in the left half-plane $\{\Re(z)<0\}$, then all solutions of (3 4) deray to 0 as $t \rightarrow \infty$.

Note that $C$ has rank one, since we have $\mathcal{X}(w)=\left(w_{1}, w_{2}, w_{3}, w_{1} \tilde{h}_{4}\right)^{T}\left(H-H_{\infty}\right)$ and

$$
C=\left.\frac{\partial H}{\partial w}\right|_{H=H_{\infty}}=\left(\begin{array}{c}
w_{1} \\
w_{2} \\
w_{s} \\
w_{1} \tilde{h}_{4}
\end{array}\right) \cdot \nabla_{w} H
$$


The zeros of the polynomial $p(z)$ are -1 times the eigenvalues of the matrix $i\left(\omega_{1} A+\omega_{2} B\right)+\alpha C$. We know that $i\left(\omega_{1} A+\omega_{2} B\right)$ is diagonalizable with eigenvalues $i \omega U, i \omega U, i \omega(U+c)$, and $i \omega(U-c)$, where $U=\omega_{1} u+\omega_{2} v$ and $\omega^{2}=\omega_{1}^{2}+\omega_{2}^{2}$. The following is a theorem about rank one perturbations of diagonalizable matrices with multiple eigenvalues

THEOREM. Let $R$ be a (complex) matrix with a double eigenvalue $\lambda$ and two linearly independent eigenvectors associated with $\lambda$. Let $S$ have rank one. Then $R+S$ has $\lambda$ as an eigenvalue.

Proof Let $v_{1}, v_{2}$ be two hnearly independent eigenvectors associated with $\lambda$. Since $S$ has rank one, there is some nontrivial combination $a v_{1}+b v_{2}$ such that $S\left(a v_{1}+b v_{2}\right)=0$. Then $(R+S)\left(a v_{1}+b v_{2}\right)=\lambda\left(a v_{1}+b v_{2}\right)$

Applying the theorem with $R=\imath\left(\omega_{1} A+\omega_{2} B\right)$ and $S=\alpha C$, we see that $s\left(\omega_{1} A+\omega_{2} B\right)+\alpha C$ has an elgenvalue $i \omega U$. This leads to the conclusion that enthalpy damping cannot produce a system (3.4) which has solutions that decay to 0 as $t \rightarrow \infty$. This result is very general; it applies to any form of enthalpy damping for which the enthalpy damping term is proportional to the difference between the local enthalpy and the freestream enthalpy Indeed, if the enthalpy damping term in (32) is formulated as $K=\left(H-H_{\infty}\right)\left(\beta_{1}(w), \beta_{2}(w), \beta_{3}(w), \beta_{1}(w)\right)^{T}$, then the matrix $C$ has rank one.

We are going to further study the system of equations with enthalpy damping, to investigate the behavior of the eigenvalues that do move off the imagnary axis and, to find a reasonable choice for $\tilde{h}_{4}$ To do this, it is not convenient to work with the matrices $A$ and $B$, for the entries of these matrices are complicated functions of $\left(w_{1}, w_{2}, w_{3}, w_{4}\right)$ It is easier to work with simlarity transforms $N^{-1} A N$ and $N^{-1} B N$, where $N$ is the matrix $\partial w / \partial \tilde{w}$ and $\tilde{w}=(\rho, u, v, p)$. This simply amounts to writing the Euler equatıons in primitive varıables rather than in conservatıve varıables One has

$$
\begin{aligned}
N & =\left(\begin{array}{cccc}
1 & & \\
u & \rho & & \\
v & 0 & \rho & \\
\left(u^{2}+v^{2}\right) / 2 & \rho u & \rho v & 1 /(\gamma-1)
\end{array}\right) \\
N^{-1} & =\left(\begin{array}{cccc}
1 & & & \\
-u / \rho & 1 / \rho & & \\
-v / \rho & 0 & 1 / \rho & \\
(\gamma-1)\left(u^{2}+v^{2}\right) / 2 & (1-\gamma) u & (1-\gamma) v & \gamma-1
\end{array}\right)
\end{aligned}
$$

The matrices $N^{-1} A N$ and $N^{-1} B N$ have been given in Warming and Beam (Ref. 14) They are

$$
N^{-1} A N=\left(\begin{array}{cccc}
u & \rho & 0 & 0 \\
0 & u & 0 & 1 / \rho \\
0 & 0 & u & 0 \\
0 & \rho c^{2} & 0 & u
\end{array}\right), \quad N^{-1} B N=\left(\begin{array}{cccc}
v & 0 & \rho & 0 \\
0 & v & 0 & 0 \\
0 & 0 & v & 1 / \rho \\
0 & 0 & \rho c^{2} & v
\end{array}\right)
$$

The matrux $N^{-1} C N$ turns out to be

$$
N^{-1} C N=\left(\begin{array}{cccc}
c^{2} /(1-\gamma) & \rho u & \rho v & \gamma /(\gamma-1) \\
0 & 0 & 0 & 0 \\
0 & 0 & 0 & 0 \\
<\text { first row times }(1-\gamma)\left(u^{2}+v^{2}-2 \tilde{h}_{1}\right) / 2>
\end{array}\right)
$$

Since $p(z)=\operatorname{det}\left(z I+z\left(\omega_{1} N^{-1} A N+\omega_{2} N^{-1} B N\right)+\alpha N^{-1} C N\right)$, we can use the expressions for $N^{-1} A N, N^{-1} B N$, and $N^{-1} C N$ to compute $p(z)$. It turns out that with $U=\omega_{1} u+\omega_{2} v$ and $\omega^{2} .=\omega_{1}^{2}+\omega_{2}^{2}$ we have $p(z)=p_{0}(z)+\alpha q(z)$ where

$$
\begin{aligned}
p_{0}(z)= & (z+i \omega U)^{2}(z+i \omega(U+c))(z+i \omega(U-c)) \\
g(z)= & \gamma\left(\tilde{h}_{4}-E\right) z^{3}+i \omega U\left((2 \gamma+1)\left(\tilde{h}_{1}-E\right)-c^{2} / \gamma\right) z^{2} \\
& \left.\omega^{2} \mid \frac{c^{2}}{\gamma^{2}}\left(2 U^{2}-c^{2}\right)+\frac{\left(\tilde{h}_{4}-E\right)}{\gamma}\left(c^{2}-(\gamma+2) U^{2}\right)\right] z \\
& -i \omega^{3} U\left(U^{2}-c^{2}\right)\left(\tilde{h}_{4}-E-\frac{c^{2}}{\gamma}\right)
\end{aligned}
$$


The polynomial $p_{0}(z)$ is the polynomial one would obtain with no enthalpy damping; its roots are purely imaginary, which shows that the Euler equations without enthalpy damping are a hyperbolic system. (To show hyperbolicity, one also has to verify that there are linearly independent eigenvectors associated with the double zero at $z=-i \omega U$.) It can be verified that $q(-i \omega U)=0$; this is a consequence of the theorem stated above.

We can obtain further information. First, take $U=c M$ and replace $z$ by cws. Next, take $\tilde{h}_{4}-E=k c^{2} / \gamma$. Note that for $k=0$ we have $\tilde{h}_{4}=E$ and for $k=1$ we have $\tilde{h}_{4}=H$. The polynomial $p(z)$ becomes

$$
\begin{aligned}
\omega^{4} c^{4}\left[(\zeta+i M)^{2}(\zeta+i(M+1))(\zeta+i(M-1))\right. \\
+\frac{\alpha c}{\omega}\left\{k \varsigma^{3}+\frac{i}{\gamma} M(2 k \gamma+k-1) \varsigma^{2}\right. \\
\left.\left.\quad+\frac{1}{\gamma}\left((2-2 k-k \gamma) M^{2}+k-1\right) \zeta-\frac{i}{\gamma}(k-1) M\left(M^{2}-1\right)\right\}\right]
\end{aligned}
$$

This factors as

$$
\begin{aligned}
\omega^{4} c^{4}(\zeta+i M)\left\{\zeta^{3}+3 i M \zeta^{2}\right. & +\left(1-3 M^{2}\right) \zeta+i M\left(1-M^{2}\right)+\frac{\alpha c}{\omega}\left[k \zeta^{2}+i \frac{M}{\gamma}(\gamma k+k-1) \zeta+\frac{k-1}{\gamma}\left(1-M^{2}\right) \mid\right\} \\
& =: \omega^{4} c^{4}(\varsigma+i M) R(\varsigma)
\end{aligned}
$$

(The polynomial $R(S)$ is exactly the polynomial one would obtain by analyzing enthalpy damping applicd to the Euler equations in one space dimension.) The plan now is to study the location of the zeros of the polynomial $R(\varsigma)$. Assume $\alpha>0$ Writing $R(\varsigma)=S(\varsigma)+(\alpha c / \omega) T(\zeta)$, we note that for $\zeta$ on the imaginary axis, $S(\zeta)$ is purely imaginary and $T(\varsigma)$ is real, bence $R$ cannot vanish on the imagnary axis unless $S$ and $T$ have a common zero on the umaginary axis. The zeros of $S$ he on the imaginary axis and are $-i M,-i(M+1),-i(M-1)$ At these $\zeta, T(\zeta)$ takes on the values $(k-1) / \gamma,-(M+1)(\gamma k-k+1) / \gamma$, and $(M-1)(\gamma k-k+1) / \gamma$, respectively. Thus if $M^{2} \neq 1$ and $k \neq 1, R$ has no zeros on the imaginary axis Hence in this case we may choose any convenient value of $M$ and use the algorithm given by J J. H. Miller (Ref. 15) for deciding whether a polynomial has all its roots in the open left half-plane.

The algorithm is as follows For any function $p(z)$, define $p^{*}(z)=\overline{p(-\bar{z})}$ Given a polynomial $p(z)$, take a point $\zeta$ in the left half-plane for which $p(z) \neq 0 \neq p^{*}(z)$, and define

$$
\not p(z)=\frac{p^{*}(\varsigma) p(z)-p(\varsigma) p^{*}(z)}{z-\varsigma}
$$

Then $\not p(z)$ is a polynomial of degree less than $p(z)$ The theorem is that $p(z)$ has all its zeros in the open left half-plane if and only if $\left|p^{*}(\zeta)\right|>|p(\zeta)|$ and $\not p$ has all its zeros in the open left half-plane Thus one can recursively apply this theorem, the degree of the polynomial decreasing at each step

Consıder the case $M^{2}<1$. In this case $M=0$ is a convenient value to pick, and one can show (using Miller's algorithm) that $R$ has all its zeros in $\{\Re(\zeta)<0\}$ if and only if $k>1$. Now consider the case $M^{2}>1$ In this case one can choose $M=2$ (for example), the result is that $R$ never has all its zeros in the open left half-plane Nor consider the case $M^{2}=1$ In this case $R$ bas a zero at $\zeta=0$, and one can show that the other zeros of $R$ are in the open left half-plane if and only if $k>1$. In summary, the polynomial $R(s)$ has all its zeros in the open left half-plane $\{\Re(\varsigma)<0\}$ if and only if $M^{2}<1$ and $k>1$ Note that the condition $M^{2}<1$ is $\left(\omega_{1} u+\omega_{2} v\right)^{2}<c^{2}\left(\omega_{1}^{2}+\omega_{2}^{2}\right)$, which holds for all $\left(\omega_{1}, \omega_{2}\right)$ if and only if $u^{2}+v^{2}<c^{2}$, i e the flow is subsonic It is interesting to note further that $k=0$ (unstable) is the case $\tilde{h}_{4}=E$, the formulation of enthalpy damping we obtained in section 2 from the potential equation with enthalpy damping, while the case $k=1$ (neutrally stable) is the same as $\tilde{h}_{4}=H$, the formulation that "can lead to instability" according to Jameson, Schmidt, and Turkel (Ref 1)

Thus enthalpy damping leads to a differential system which has a general solution that does not decay to zero Of the four eigenvalues on the imaginary axis when there is no enthalpy damping, one stays on the imaginary axis when enthalpy damping is introduced and three move off the imaginary axis The analysis suggests that the energy equation with enthalpy damping be written in the form

$$
(\rho E)_{1}+(\rho u H)_{x}+(\rho v H)_{y}+\alpha \rho\left(E+k c^{2} / \gamma\right)\left(H-H_{\infty}\right)=0
$$

The eigenvalues that move of the imaginary axis move into the left half-plane provided that $k>1$ and $u^{2}+v^{2}<c^{2}$ 
It is appropriate here to comment on the missing factor in the analysis, the dissipation term Going back to (1.7), take a dissipation term of the form

$$
D(w)=\left(\partial_{x}^{1}+\partial_{y}^{4}\right)\left(w_{1}, w_{3}, w_{3}, \rho H\right)^{T}
$$

The point here is that the fourth component is not $w_{1}$, it is instead $\rho H$. This is done because the system of equations with artificial dissipation still admits steady solutions with $H=H_{\infty}$. To see this, note that when the continuity equation with dissipation is multipled by $H_{\infty}$ and subtracted from the energy equation with dissipation we obtain

$$
\begin{aligned}
\left(\rho\left(E-H_{\infty}\right)\right)_{t} & +\left(\rho u\left(H-H_{\infty}\right)\right)_{x}+\left(\rho v\left(H-H_{\infty}\right)\right)_{y} \\
& +\alpha \rho\left(\tilde{h}_{4}-H_{\infty}\right)\left(H-H_{\infty}\right)+\epsilon\left(\partial_{x}^{4}+\partial_{y}^{4}\right) \rho\left(H-H_{\infty}\right)=0
\end{aligned}
$$

which evidently admits steady solutions with $H=H_{\infty}$. We would want to look at the polynomial

$$
p(z)=\operatorname{det}\left(z I+i\left(\omega_{1} A+\omega_{2} B\right)+\alpha C+\epsilon\left(\omega_{1}^{4}+\omega_{2}^{4}\right) D\right)
$$

where $D=\partial D / \partial w$. If we do this and then form $N^{-1} D N$, we find

$$
N^{-1} D N=\left(\begin{array}{cccc}
1 & 0 & 0 & \\
0 & 1 & 0 & 0 \\
0 & 0 & 1 & 0 \\
(\gamma-1)^{2}\left(u^{2}+v^{2}\right) / 2 & 0 & 0 & \gamma
\end{array}\right)
$$

The fact that the fourth row here is not the fourth row of the identity matrix has the consequence that $p(z)$ with $\epsilon \neq 0$ is vastly more complicated than $p(z)$ with $\epsilon=0$ This author has not been able to make any headway in analyzing the case $\epsilon \neq 0$ because of the formidable algebraic difficultıes In fact, even for the case of no inthalpy damping $(\alpha=0)$ this author is not aware of any proof of stability of the linearized system with dissipation, because the dissipation term is applied to a nonlinear function of $w$

Some experiments were done with the code FLO52R in changing the form of the enthalpy damping term in the energy equation The form $\tilde{h}_{4}=E+k c^{2} / \gamma$ was used, and various values of $k$ were tried Only one line of the code was changed The first case was flow about a NACA0012 aurfoll at a freestream Mach number 08 and at an angle of attack of $125^{\circ}$ The code was run in multigrid mode with 40 cycles on a $48 \times 8$ mesh, then 40 cycles on a $96 \times 16$ mesh, and finally 100 cycles on a $192 \times 32$ mesh. A five-stage scheme with coefficients $(1 / 4,1 / 6,3 / 8,1 / 2,1)$ was used, with two dissipation evaluations per time step For the unmodified code, the convergence rate for 100 cycles on the $102 \times 32$ grid was 09557 A table of convergence rate vs $k$ for the modified code is shown here

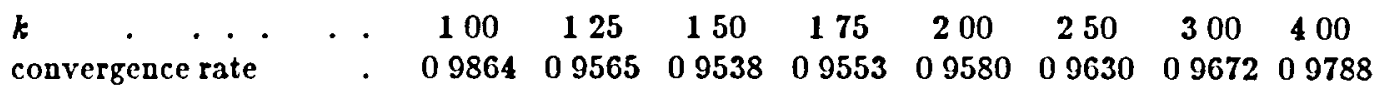

Evidently the optımum $k$ is approxımately 15 For a subcritıcal case with a freestream Mach number of 063 and an angle of attack of $2^{\circ}$, with all other parameters the same, the unmodified code had convergence rate 09288 The modified code produced the following results.

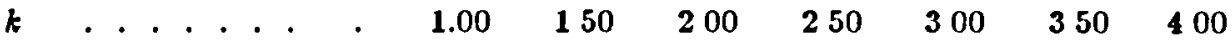

$$
\begin{aligned}
& \begin{array}{lllllllll}
\text { convergence rate } & \cdots & 09451 & 0.9303 & 09282 & 09276 & 09273 & 0.9275 & 09302
\end{array}
\end{aligned}
$$

Here the optimal $k$ is apparently between 25 and 35 In both cases it is clearly difficult to improve on the original code This author believes the excellent convergence behavior of the unmodiffed code may be explaned as follows.

The energy equation enthalpy damping term in the unmodified code comes from discretizing

$$
\partial_{t}\left(\rho\left(E-H_{\infty}\right)\right)+\partial_{x}\left(\rho u\left(H-H_{\infty}\right)\right)+\partial_{y}\left(\rho v\left(H-H_{\infty}\right)\right)+\alpha \rho\left(H-H_{\infty}\right)=0
$$

$3 \mathrm{e}$, this equation is used in place of the fourth equation of (29). In terms of the partial diferential equation system, an equivalent equation is obtained by multiplyıng the first equation of (29) by $H_{\infty}$ and adding to this, obtaining

$$
\partial_{t}(\rho E)+\partial_{x}(\rho u H)+\partial_{y}(\rho v H)+\alpha \rho\left(H-H_{\infty}\right)\left(H_{\infty}+1\right)=0
$$


In the notation of (3 2), $\tilde{h}_{4}=H_{\infty}+1$. With the definition of $\tilde{h}_{4}$ as $\tilde{h}_{4}=E+k c^{2} / \gamma$ we would obtain $\tilde{h}_{4}=H_{\infty}+1$ If we had $k=\gamma\left(E_{\infty}-E+c_{\infty}^{2} / \gamma+1\right) / c^{2}$. Now, the nondimensionalization in the FLO52 codes is such that $c_{\infty}^{2}=\gamma$, hence at freestream conditions we would have $k=2$. Thus $\tilde{h}_{1}=E+2 c^{2} / \gamma$ is roughly the same as the unmodified code; also the previously noted experiments showed $k=15$ in one case and $k \in(25,35)$ in the other case were near-optimal. Thus $k=2$ (nearly the original code) would be expected to give excellent results It is also clear that the unmodıfied code is equivalent to a code with locally varying $k$; this author has not experimented with other forms of locally varying enthalpy damping terms.

\section{Finite Difference Formulation with Enthalpy Damping}

The anm of this section is to study the actual finite difference implementation of enthalpy damping. The aim is to understand why enthalpy damping can be effective in practice when on the level of the partial differential equation it does not produce a system with solutions which tend to zero.

The general formulation of enthalpy damping to be studied is as follows. First spatial differencing is appled, sending $w^{n}$ to $\widetilde{w^{n}}:=\mathcal{F}\left(w^{n}\right)$. Then enthalpy damping is applied, via

$$
w^{n+1}-\widetilde{w^{n}}+\alpha \Delta t K\left(w^{n+1}, \widetilde{w^{n}}\right)=0
$$

For example, the first equation could be taken to be

$$
\rho_{i j}^{n+1}-\widetilde{\rho_{i j}^{n}}+\alpha \Delta t p_{i j}^{n+1}\left(\widetilde{H_{i j}^{n}}-H_{\infty}\right)=0
$$

Notice that in this formulation (the FLO52 formulation) the enthalpy damping term is in a sem-implicit form which is readily solved for $\rho_{1,}^{n+1}$. If the enthalpy damping term were fully implicit, it would be nonlinear in $w^{n+1}$ and difficult to solve

We plan to study the stability of (4 1$)$ about a solution $w^{*}$ with $w^{*}=\mathcal{F}\left(w^{*}\right)$ and $H\left(w^{*}, w^{*}\right)=0$. Perturbing to $w^{*}+\epsilon u$, we find the perturbation equation is

$$
w^{n+1}-A w^{n}+\alpha \Delta t\left(B_{1} w^{n+1}+B_{2} A w^{n}\right)=0
$$

where

$$
\begin{array}{ll}
A & =D F\left(w^{*}\right) \quad \text { (the Jacobian matrix) } \\
B_{1}=D_{1} H\left(w^{*}, w^{*}\right) & \text { (Jacobian with respect to first argument) } \\
B_{2}=D_{2} H\left(w^{*}, w^{*}\right) & \text { (Jacobian with respect to second argument) }
\end{array}
$$

The equation (42) is equivalent to

$$
\left(I+\alpha \Delta t B_{1}\right) w^{n+1}=\left(I-\alpha \Delta t B_{2}\right) A w^{n}
$$

Let us take

$$
\mathcal{H}\left(w^{n+1}, \widetilde{w^{n}}\right)=\left(\widetilde{H^{n}}-H_{\infty}\right) \mathcal{H}^{\prime}\left(w^{n+1}, \widetilde{w^{n}}\right)
$$

Then $B_{1}=0$ and $B_{2}=H^{\prime}\left(w^{*}, w^{*}\right) \cdot \nabla_{\widetilde{w^{n}}}\left(\widetilde{H^{n}}-H_{\infty}\right)$. Furthermore, if the basic iteration scheme is an explicit multistage method with central spatial differencing, then $\mathcal{f}\left(w^{n}\right)=P(\Delta t L) w^{n}$, where $P$ is a polynomial, $L=$ $A \delta_{x} / 2 \Delta x+B \delta_{y} / 2 \Delta y$, and $\delta_{x}$ and $\delta_{y}$ are central space difference operators Thus the matrix problem (43) becomes

$$
w^{n+1}=\left(I-\alpha \Delta t B_{2}\right) P(\Delta t L) w^{n}
$$

Fourier transforming in space produces the problem

$$
\widehat{w}^{n+1}=\left(I-\alpha \Delta t B_{2}\right) P(\Delta t \hat{L}) \widehat{w}^{n}
$$

where $\Delta l \hat{L}=i\left(\omega_{1} A+\omega_{2} B\right)$ and $\omega_{1}=\Delta t \sin \theta / \Delta x, \omega_{2}=\Delta t \sin \phi / \Delta y$, and $\theta, \phi$ are the Fourier dual variables. 


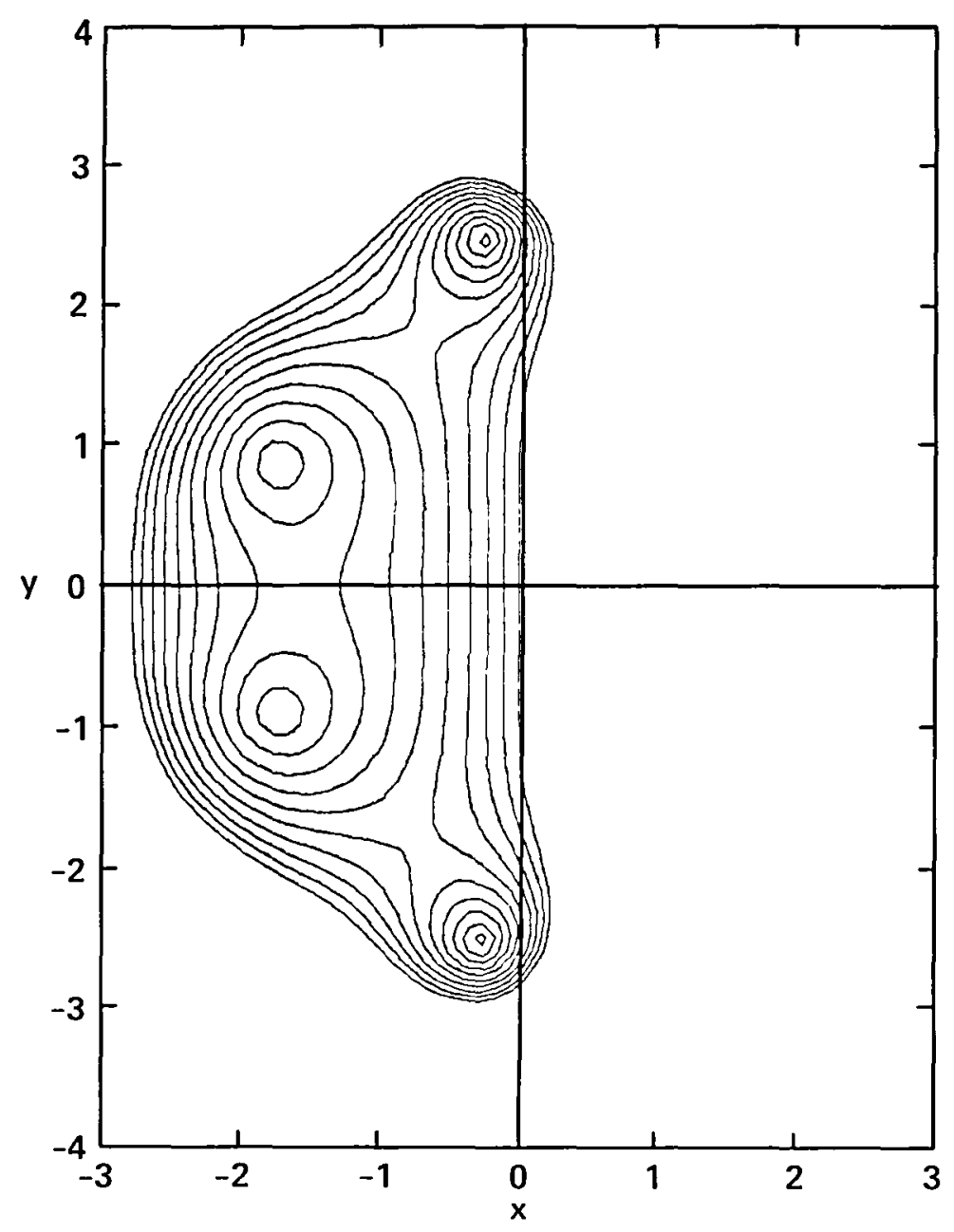

Figure 41 . Contours of $|P(z)|$ in the complex plane for classical fourth-order Runge-Kutta. Contour levels: $01,0.2,03, \ldots, 10$.

A reasonable form (consistent with the work of section 3 ) for the implementation of the enthalpy damping step is

$$
\begin{array}{r}
\rho^{n+1}-\widetilde{\rho^{n}}+\alpha \Delta t \rho^{n+1}\left(\widetilde{H^{n}}-H_{\infty}\right)=0 \\
(\rho u)^{n+1}-\left(\widetilde{\rho u)^{n}}+\alpha \Delta t(\rho u)^{n+1}\left(\widetilde{H^{n}}-H_{\infty}\right)=0\right. \\
(\rho v)^{n+1}-\left(\widetilde{\rho v)^{n}}+\alpha \Delta t(\rho v)^{n+1}\left(\widetilde{H^{n}}-H_{\infty}\right)=0\right. \\
(\rho E)^{n+1}-\left(\widetilde{\rho E)^{n}}+\alpha \Delta t \mid(\rho E)^{n+1}+k \widetilde{p^{n}}\right)\left(\widetilde{H^{n}}-H_{\infty}\right)=0
\end{array}
$$

The parameter $k$ here is the same as in the previous section Then our perturbation equation is

$$
w^{n+1}=\left(I-\alpha \Delta t B_{2}\right) P(\Delta t \hat{L})
$$

For the decay of perturbations about $w^{*}$, it is necessary and sufficient that all eigenvalues $\lambda$ of $\left(I-\alpha \Delta t B_{2}\right) P(\Delta t \hat{L})$ satisfy $|\lambda|<1$ Furthermore, the asymptotic rate of decay is given by the modulus of the largest eigenvalue. For $\alpha=0$ (no enthalpy damping) we recover the usual CFL-like condition $\max ,\left|P\left(\lambda_{3}\right)\right|<1$, where $\lambda_{\text {, }}$ are the eigenvalues of $\Delta t \hat{L}: \lambda_{1}=\lambda_{2}=i \omega U, \lambda_{3}=i \omega(U+c), \lambda_{1}=i \omega(U-c)$, where $\omega U:=\omega_{1} u+\omega_{2} v$ and $\omega^{2}:=\omega_{1}^{2}+\omega_{2}^{2}$.

For example, the explicit Euler method has $P(\Delta t \hat{L})=I-\Delta t \hat{L}$ (this is unstable); the classical fourth-order Runge-Kutta method has $P(x)=1-x+x^{2} / 2-x^{3} / 6+x^{4} / 24$, for which it is known that the stability condition $\max ,|P(\lambda)|<$,1 becomes $\max ,|\lambda|<,2 \sqrt{2}$. Contours of $|P(z)|$ in the complex $z$-plane are shown in Figure 41 for the fourth-order Runge-Kutta method. 
The question before us now is, does the modulus of the maximum eigenvalue decrease when $\alpha \Delta t$ is increased from 0 ? Also, what are good values for $\alpha \Delta t$ and $k$ ? For $\mathcal{H}$ as in (4.4) we have

$$
B_{2}=\left(\begin{array}{c}
\rho \\
\rho u \\
\rho v \\
\rho E+k p
\end{array}\right) \cdot \frac{1}{\rho}\left(-\gamma E+(\gamma-1)\left(u^{2}+v^{2}\right),(1-\gamma) u,(1-\gamma) v, \gamma\right)
$$

Again we have a matrix of rank one, $B_{2}$, and a diagonalizable matrx $P(\Delta t \hat{L})$ with a double eigenvalue at $P(i \omega U)$, so the theorem of the previous section implies that $\left(I-\alpha \Delta t B_{2}\right) P(\Delta t \hat{L})$ has as one of its eigenvalues $P(\imath \omega U)$. Thus If this elgenvalue is the eigenvalue of maximum modulus, then enthalpy damping will not improve the convergence rate of the iteration. On the other hand, if one of the other eigenvalues is obstructing the convergence, then enthalpy damping may help.

To help in studying this question, it is useful to diagonalze the matrix $\widehat{L}$. The matrix $T$ which diagonalizes $\omega_{1} A+\omega_{2} B$ is known analytically (see Warming and Beam (Ref 14)). It is

$$
T=\left(\begin{array}{cccc}
1 & 0 & \rho /(\sqrt{2} c) & \rho / \sqrt{2} c \\
0 & \omega_{2} / \omega & \omega_{1} /(\sqrt{2} \omega) & -\omega_{1} /(\sqrt{2} \omega) \\
0 & -\omega_{1} / \omega & \omega_{2} /(\sqrt{2} \omega) & -\omega_{2} /(\sqrt{2} \omega) \\
0 & 0 & \rho c / \sqrt{2} & \rho c / \sqrt{2}
\end{array}\right)
$$

Then

$$
\begin{aligned}
T^{-1}\left(I-\alpha \Delta t B_{2}\right) P(\Delta t \hat{L}) T= & \left(I-\alpha \Delta t T^{-1} B_{2} T\right) T^{-1} P(\Delta t \hat{L}) T \\
= & \left(I-\alpha \Delta t T^{-1} B_{2} T\right) \\
& \cdot \operatorname{diag}\{P(i \omega U), P(i \omega U), P(i \omega \mid U+c]), P(i \omega \mid U-c])\} .
\end{aligned}
$$

The characteristic polynomal of this matrix vanıshes at $P(s \omega U)$, and upon division by $z-P(i \omega U)$ the characteristic polynomal becomes (writing $\sigma=P(i \omega U), \sigma_{+}=P(\imath \omega|U+c|), \sigma_{-}=P(i \omega[U-c \mid)$, and $M=U / c)$,

$$
\begin{aligned}
& (z-\sigma)\left(z-\sigma_{+}\right)\left(z-\sigma_{-}\right) \\
& +\frac{\alpha \Delta l c^{2}}{2 \gamma}\left[z^{2}\left\{2(k-1) \sigma+(1+M)(\gamma k-k+1) \sigma_{+}+(1-M)(\gamma k-k+1) \sigma_{-}\right\}-\right. \\
& -z\left(\{\gamma k(1+M)+(k-1)(1-M)\} \sigma \sigma_{+}+\{\gamma k(1-M)+(k-1)(1+M)\} \sigma \sigma_{-}\right. \\
& \left.\quad+2(\gamma k-k+1) \sigma_{+} \sigma_{-}\right) \\
& \left.\quad+2 \gamma k \sigma \sigma_{+} \sigma_{-}\right\}
\end{aligned}
$$

Note that $\alpha$ has units of time $e^{-1}$ velocity ${ }^{-2}$ (cf. (4 4)), so $\alpha \Delta t c^{2}$ is dimensionless.

We will study the roots of the polynomial in (45) numerically. Fux a "Mach number" $M=U / c$. Define $\nu$ ("CFL number") as $\max (|\omega U|,|\omega(U+c)|,|\omega(U-c)|)$. Then

$$
\omega U= \begin{cases}\nu \operatorname{sgn}(M) / \max (|1+1 / M|,|1-1 / M|), & \text { if } M \neq 0 \\ 0 & \text { if } M=0\end{cases}
$$

Now for a given $M$ we can graph the spectral radius vs $\nu$ by using the definition of $\omega U$ from (46) and then numerically finding the roots of the polynomial (45). We can further search for an optimal value of the dimensionless parameter $\alpha \Delta t c^{2}$ by using as optımality criterion the integral between the curves of spectral radius vs. $\nu$ for $\alpha \Delta t c^{2}=0$ and $\alpha \Delta t c^{2} \neq 0$, and searching for $\alpha \Delta t c^{2}$ which maxumizes this integral (Numerically, the composite trapezoldal rule was used for the integration.) Two typical graphs of spectral radus vs. $\nu$ without and with enthalpy damping are shown in Figure 42 Note that enthalpy damping has little or no effect at small CFL numbers, it is only for CFL numbers near the stability limit that enthalpy damping is significantly helpful For supersonic flow $(M>1)$, the spectral radius with enthalpy damping was observed to be greater than 1 . In all subsonic cases investigated, the spectral raduus with enthalpy damping was exactly the same as or slightly less than the spectral radus without enthalpy 


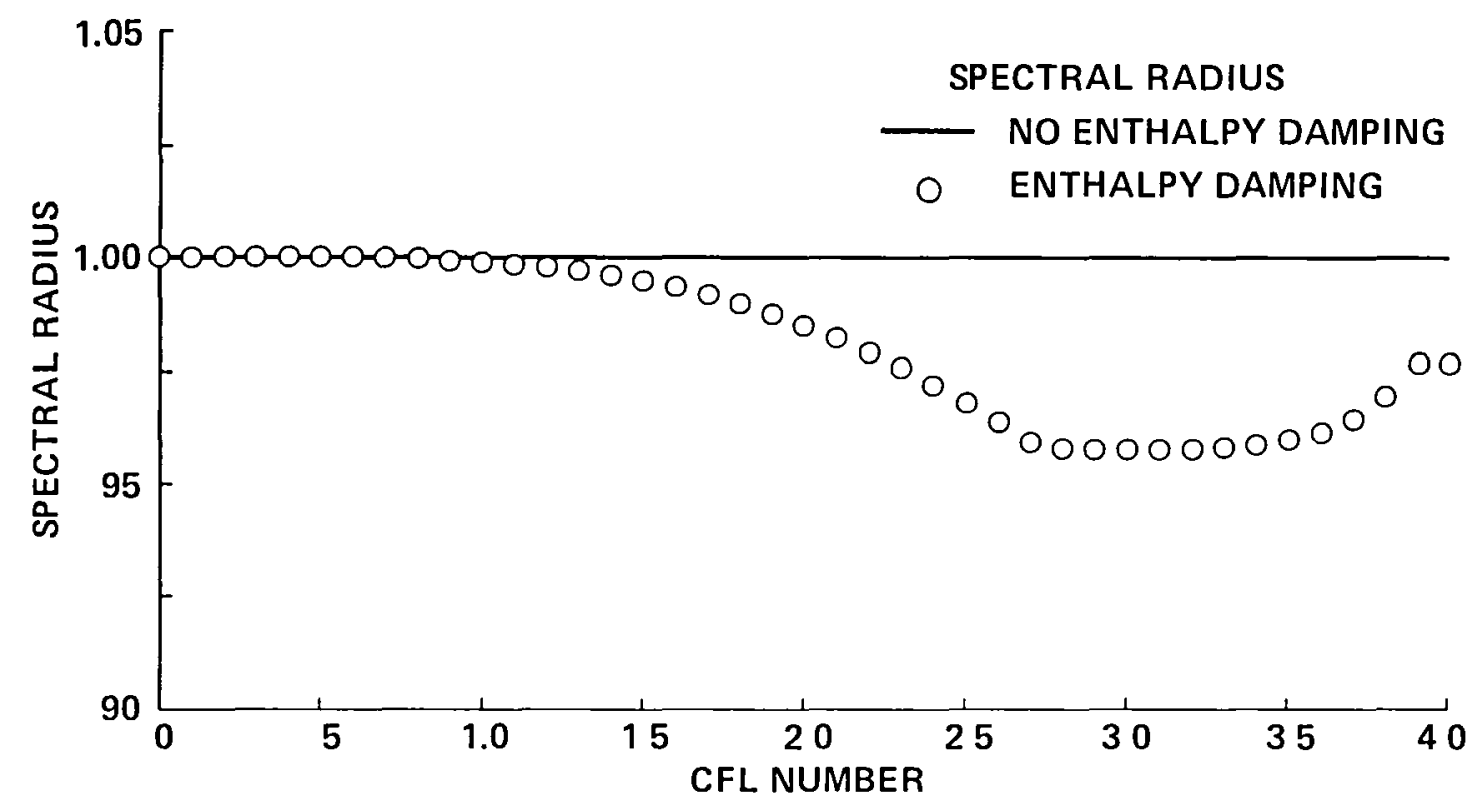

Figure 42 (a). Spectral radius vs. CFL number for numerical scheme without and with enthalpy damping. Five-stage method with coefficients $(1 / 4,1 / 6,3 / 8,1 / 2,1), M=08, k=2, \alpha \Delta t c^{2}=025$

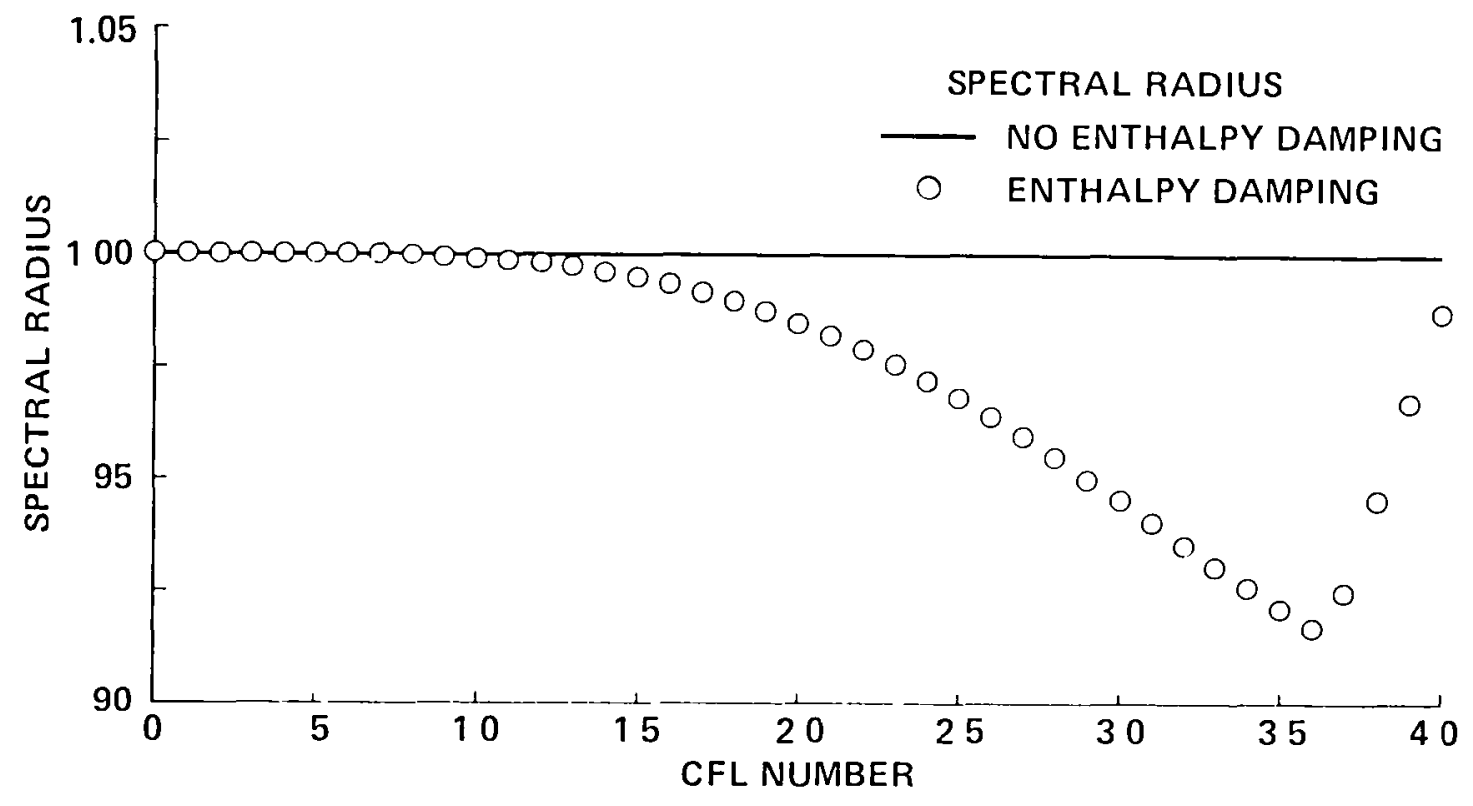

Figure 4 2(b). Same as (a) except $\alpha \Delta t c^{2}=05$.

damping, at least in the region of stability The five-stage method with coefficients $(1 / 4,1 / 6,3 / 8,1 / 2,1)$ was studied, with $0 \leq \nu \leq 4$ (the stability limit) With $k=2$ and two different Mach numbers, $M=08$ and $M=04$, the following results were obtained for the area between the curves as a function of $\alpha \Delta t c^{2}$.

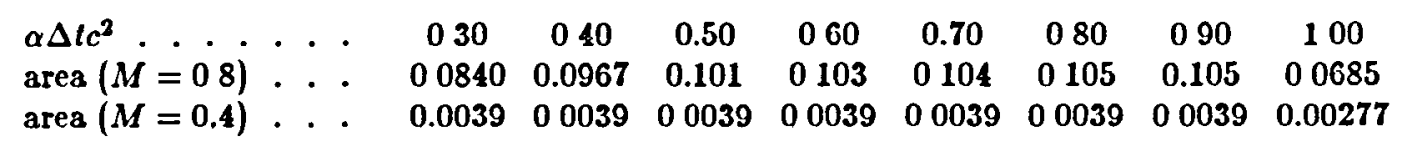


Evidently there is not a sharp optimum value of $\alpha \Delta t c^{2}$, for in the first case any $\alpha \Delta t c^{2}$ between 05 and 09 gives approximately the same result, while in the second case any $\alpha \Delta t c^{2} \in(0.3,0.9)$ can be chosen. If the speed of sound is normalized to be $\gamma$ at infinity (as in the FL052 codes), then our good range for $\alpha \Delta t$ becomes $\alpha \Delta t \in(036,064)$ in the first case, and $\alpha \Delta t \in(0.21,0.64)$ in the second case.

\section{Conclusions}

The Euler equations with enthalpy damping can be studied as a system of partial differential equations. Linear stability analysis of the system around a state with constant enthalpy shows that not all solutions decay to zero. The analysis suggests that the enthalpy damping term in the energy equation be written as $\alpha \rho\left(E+k c^{2} / \gamma\right)\left(H-H_{\infty}\right)$. For this formulation, the linear stability analysis shows stability (neutral stablity, $i$ e., no eigenvalues in the open right half plane) if $k \geq 1$ and the flow is subsonic.

When the equations are discretized via an explicit multistage method, the spectral radius can be decreased by enthalpy damping, even though it is again the case that one of the eigenvalues is unchanged. A numerical study with $k=2$ revealed that $\alpha \Delta t c^{2} \approx 05$ should give good results The particular value of $\alpha \Delta t$ this leads to depends on the nondımensionalization of a given computer code

This analysis has not taken the dissipation term into account, because the dissipation operator in the energy equation is applied to $\rho H$, not $\rho E$ (so that the equations with dissipation will still have constant enthalpy at steady state), which makes the analysis much more complicated All the analysis shows that enthalpy damping should be destabilizing for supersonic flow, but in practice enthalpy damping does not harm the convergence for the FLO52 codes in the transonic range. It is probably the case that the dissipation term is effective in stablizing the computation, even in locally supersonic regions of flow

\section{References}

1 Jameson, A , Schmidt, W, and Turkel, E , Numerical Solutions of the Euler Equations by Finite Volume Methods Using Runge-Kutta Time-Stepping Schemes, AIAA paper 81-1259, AIAA 14th Fluid and Plasma Dynamics Conference, Palo Alto, 1981

2. Jameson, A, Solution of the Euler Equations for Two-Dimensional Transonic Flow by a Multigrid Method, Appl. Math and Computation 13, no 3-4, pp 327-355, 1983

3 Ruzzı, A, Damped Euler-Equation Method to Compute Transonic Flow A round Wing-Body Combınations, AIAA J. 20, 1321-1328, 1982

4 Pulliam, T H., Euler and Thin Layer Navier-Stokes Codes. ARC2D, ARC9D, in Computatsonal Fluid Dynamics Users' Workshop, University of Tennessee Space Institute Publication No E02-4005-023-84, March 1984

5 Lerat, A , Sidès, J , and Daru, V., An Implscst Fınste.Volume Method for Solving the Euler Equatıons, in Erghth International Conference on Numencal Methods in Flund Dynamics, Springer Lecture Notes in Physics no 170, ed E Krause, Springer-Verlag, 1982

6 Johnson, G, Multiple Grid Acceleration of Viscous and Inviscud Flow Computations, Appl Math and Computation 13, no. 3-4, pp. 327-355, 1983.

7 N1, R-H, A Multiple Grad Scheme for Solving the Euler Equations, AIAA Paper 81-1025, June 1981.

8 Agarwal, R. K, and Deese, J. E., Euler Solutions for Flow Past an Airfoul from Subsonic to High Supersonic Mach Numbers, AIAA 6th Computational Flud Dynamics Conference, July 1983

9 Osher, S, and Chakravarthy, S, Upwand Schemes and Boundary Conditions with Applacations to Euler Equations in General Geometries, J Comp Phys 50, pp. 447-481, 1983

10. Mulder, W., and van Leer, B, Implictt Upwind Methods for the Euler Equations, AIAA 6th Computational Fluid Dynamics Conference, July 1983

11. Buning, P G, and Steger, J. L, Solutions of the Two-Dimensional Euler Equations With Generalized Coordinate Trangformations Using Flux Vector Splitting, AlAA Paper 82-0971, St. Lous, Mo., June 1982.

12 MACSYMA Reference Manual, Laboratory for Computer Science, Massachusetts Institute of Technology, January 1983.

13. Courant, R, and Hilbert, D, Methods of Mathematical Physics, vol 2, Interscience Publishers, New York, 1962 
14. Warming, R. F., and Beam, R. M., On the Construction and Application of Implicit Factored Schemes for Conservation Laws, SIAM-AMS Proceedings, vol. 11, 1978, pp. 85-129.

15. Miller, J. J. H., On the Stability of Differential Equations, SIAM J Control 10, 1972, pp. 639-648. 


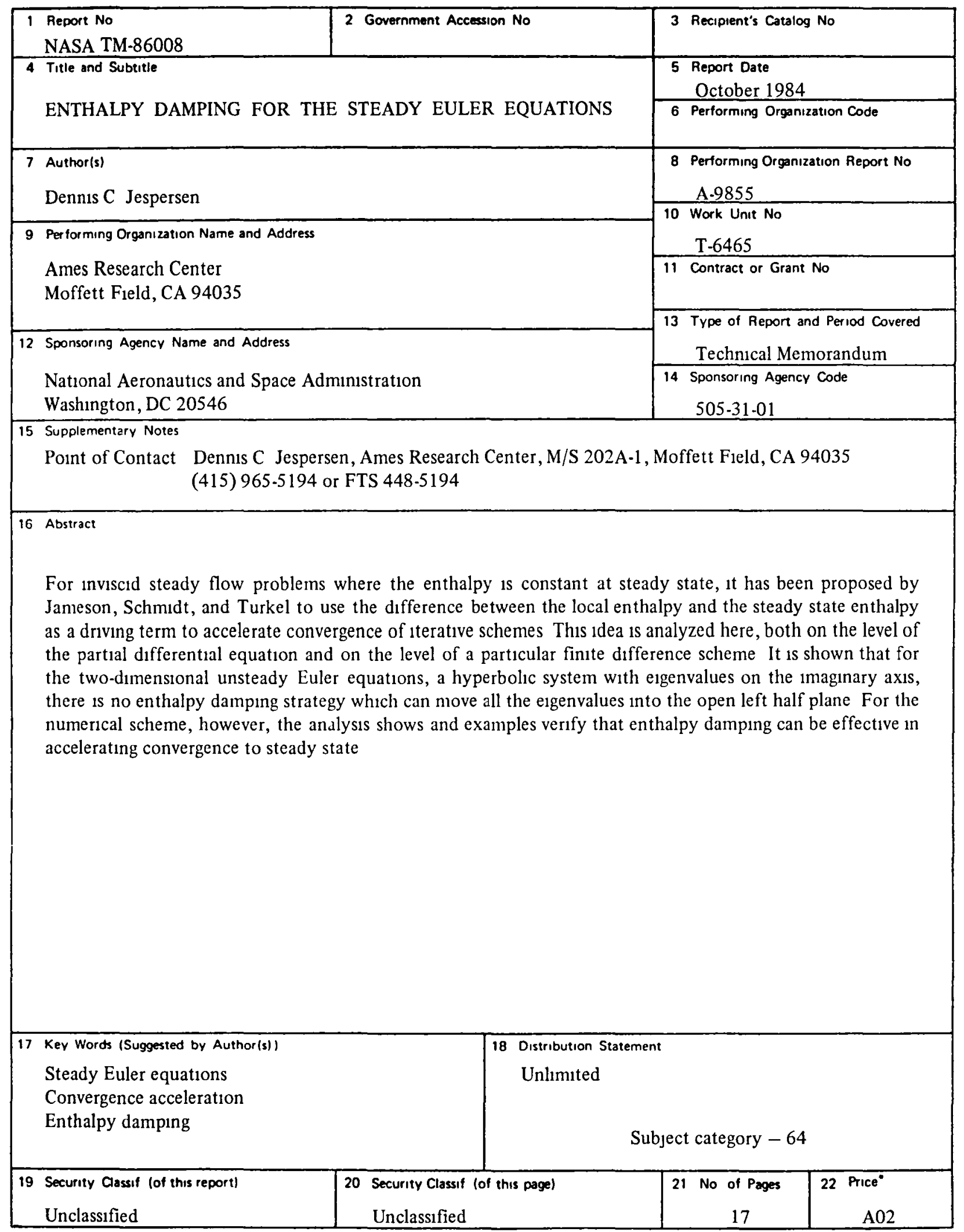

-For sale by the National Technical Information Service, Springfield. Virginia 22161 
End of Document 